PROCEEDINGS OF THE

AMERICAN MATHEMATICAL SOCIETY

Volume 128, Number 5, Pages 1443-1450

S 0002-9939(00)05671-9

Article electronically published on February 7, 2000

\title{
ON COMPLETE GRAPHS WITH NEGATIVE R-MEAN CURVATURE
}

\author{
MARIA FERNANDA ELBERT
}

(Communicated by Christopher Croke)

\begin{abstract}
We generalize Efimov's Theorem for graphs in Euclidean space using the scalar curvature, with an additional hypothesis on the second fundamental form.
\end{abstract}

\section{INTRODUCTION}

The celebrated Efimov's Theorem says that there is no complete surface with negative Gaussian curvature $K$ bounded away from zero in the Euclidean 3-space $\mathbb{R}^{3}$. This theorem has been drawing the attention of many mathematicians over the years. A generalization for higher dimensions is still being pursued and there are several possible choices of objects that can be used to replace $K$ (cf., for instance, $[\mathrm{SX}]$ and the references therein). Here, we choose the scalar curvature as a substitute for $K$ and we generalize Efimov's Theorem for graphs in $\mathbb{R}^{n+1}$, under an additional hypothesis on the second fundamental form of the graph. Let $\|A\|$ denote the norm of the second fundamental form. We prove:

Theorem 1.1. There is no complete graph in $\mathbb{R}^{n+1}$ with $\|A\|$ bounded and negative scalar curvature bounded away from zero.

We remark that the conclusion of the theorem may not hold if the hypersurface is not a graph. Indeed, there is an example due to T.Okayasu $\mathrm{O}$ of an $O(2) \times$ $O(2)$-invariant complete hypersurface of constant negative scalar curvature in $\mathbb{R}^{4}$. Clearly, this example is not a graph.

On the other hand, we do not know whether the theorem holds without the boundedness of $\|A\|$.

We prove Theorem 1.1 not only for the scalar curvature but also for all r-mean curvatures (see definitions in the preliminaries). We will actually prove a stronger version of it, namely

Theorem 1.2. There is no complete graph in $\mathbb{R}^{n+1}$ with $\left\|P_{r}\right\|$ bounded and negative $r+1$-mean curvature $H_{r+1}$ bounded away from zero, $r+1$ even.

Here, $P_{r}$ is the $r \underline{t h}$ Newton Tensor defined in Section 2. If $\|A\|$ is bounded, then $\left\|P_{r}\right\|$ is also bounded. So we could change the hypothesis that $\left\|P_{r}\right\|$ is bounded by boundedness of $\|A\|$. In the case $r=1$ these hypotheses are, in fact, equivalent.

Received by the editors June 17, 1998.

2000 Mathematics Subject Classification. Primary 53C42; Secondary 53A10.

Key words and phrases. Negative r-mean curvature, complete graphs, divergence, Cheeger constant. 
The proof of this theorem depends essentially on the fact, well known for the mean curvature, that the r-mean curvature of a graph is a divergence (see the Main Lemma 3.1).

In this context we could quote a result due to S.S.Chern (corollary of Theorem (4) of [Che] that asserts the following.

Theorem. There is no complete graph in $\mathbb{R}^{n+1}$ satisfying

(i) $H_{2} \leq-k, k=$ constant $>0$;

(ii) $\left\langle P_{1} A v, v\right\rangle \leq-\frac{1}{2} n(n-1) k|v|^{2}$, for all $v \in \mathbb{R}^{n}$.

Here, $\mathrm{H}_{2}$ is the scalar curvature of the graph and $P_{1}$ is the first Newton Tensor of the graph.

\section{Preliminaries}

Let $x: M^{n} \longrightarrow \bar{M}^{n+1}$ be an isometric immersion of an orientable connected Riemannian $n$-manifold into an oriented Riemannian $n+1$-manifold, and let $A_{p}$ : $T_{p} M \longrightarrow T_{p} M$ be the linear operator associated to the second fundamental form of $x$. Denote by $k_{1}, k_{2}, \ldots, k_{n}$ its eigenvalues, namely the principal curvatures of $x$. We consider the elementary symmetric functions of $k_{1}, k_{2}, \ldots, k_{n}$ :

$$
\begin{array}{llrl}
S_{0} & =1, & \\
S_{r} & =\sum_{i_{1}<\ldots<i_{r}} k_{i_{1}} \ldots k_{i_{r}} & & (1 \leq r \leq n), \\
S_{r}=0 & & (r>n)
\end{array}
$$

and define the $r$-mean curvature $H_{r}$ of $x$ by

$$
S_{r}=\left(\begin{array}{l}
n \\
r
\end{array}\right) H_{r} .
$$

The study of the r-mean curvatures is related to the study of the classical Newton transformations $P_{r}$ defined inductively by

$$
\begin{aligned}
& P_{0}=I, \\
& P_{r}=S_{r} I-A P_{r-1} .
\end{aligned}
$$

Each $P_{r}$ is a self-adjoint operator that has the same eigenvectors of $A$.

Let $e_{1}, e_{2}, \ldots, e_{n}$ be orthonormal eigenvectors of $A$ corresponding, respectively, to the eigenvalues $k_{1}, k_{2}, \ldots, k_{n}$. We denote by $A_{i}$ the restriction of the transformation $A$ to the subspace normal to $e_{i}$, and by $S_{r}\left(A_{i}\right)$ the r-symmetric function associated to $A_{i}$. The proof of the following lemma can be found in [BC], Lemma (2.1).

Lemma 2.1. For each $1 \leq r \leq n-1$, we have

(i) $P_{r}\left(e_{i}\right)=S_{r}\left(A_{i}\right) e_{i}$ for each $1 \leq i \leq n$.

(ii) $\operatorname{trace}\left(A P_{r}\right)=\sum_{i=1}^{n} k_{i} S_{r}\left(A_{i}\right)=(r+1) S_{r+1}$.

\section{The Main Lemma}

Let $\left(M^{n}, g\right)$ be a connected orientable Riemannian $n$-manifold with Riemannian metric $g$, and let $f: M \longrightarrow \mathbb{R}$ be a differentiable function. We denote the graph of $f$ by $\Gamma_{f}$, namely, $\Gamma_{f}=\{(p, f(p)) ; p \in M\}$, and consider its natural embedding in $M \times \mathbb{R}$, that is, $x(M)=\Gamma_{f}$, where $x$ is given by

$$
\begin{aligned}
x: M & \longrightarrow M \times \mathbb{R} \\
p & \longmapsto(p, f(p)) .
\end{aligned}
$$


In $M \times \mathbb{R}$, we consider the product metric to be denoted by $h$. We consider in $M$ the pull-back metric denoted by $x^{*} h$. Accordingly we write $(M, g)$ or $\left(M, x^{*} h\right)$ depending on which metric we are considering. We denote by $\bar{\nabla}$ the connection of $(M \times \mathbb{R}, h)$ and by $\nabla_{1}$, respectively $\nabla_{2}$, the connection of $(M, g)$, respectively the connection of $\mathbb{R}$. We use $\bar{\nabla} \phi, \nabla_{1} \phi$ or $\nabla_{2} \phi$ to indicate the gradient of a function $\phi$ in the corresponding metric.

We can see $\Gamma_{f}$ as the inverse image of the regular value 0 for the differentiable function

$$
\begin{aligned}
F: M \times \mathbb{R} & \longrightarrow \mathbb{R} \\
(p, z) & \longmapsto f(p)-z,
\end{aligned}
$$

that is, $\Gamma_{f}=F^{-1}(0)$. In this way, we can choose the unit vector field normal to $\Gamma_{f}$ to be

$$
N=\frac{\bar{\nabla} F}{|\bar{\nabla} F|}
$$

Let us fix $p \in M$ and let $\left\{e_{i}\right\}_{i=1}^{n}$ be an orthonormal frame of $(M, g)$ in a neighborhood $\mathcal{U}$ of $p$. Then, $\left\{\left(e_{1}, 0\right), \ldots,\left(e_{n}, 0\right),(0,1)\right\}$ is an orthonormal frame of $M \times \mathbb{R}$ in the neighborhood $\mathcal{U} \times \mathbb{R}$. We write $E_{i}=\left(e_{i}, 0\right), i=1, \ldots, n$, and $E_{n+1}=(0,1)$. Clearly,

$$
\bar{\nabla} F=\left(\nabla_{1} f, 0\right)-E_{n+1}
$$

and, if we set $w=|\bar{\nabla} F|=\sqrt{1+\left|\nabla_{1} f\right|^{2}}$, we can write

$$
N=\frac{\bar{\nabla} F}{w}=\left(\frac{\nabla_{1} f}{w}, 0\right)-\frac{E_{n+1}}{w} .
$$

All entities associated to a given isometric immersion (e.g. $A, P_{r}, S_{r}, H_{r}$ ) will refer, in this section, to the immersion $x$ given in (10) with the chosen orientation $N$.

Main Lemma 3.1. If any of the following conditions

(i) $r=1$ and $\operatorname{Ric}_{(M, g)}=0$;

(ii) $r$ is arbitrary and $(M, g)$ is flat

holds, then

$$
-(r+1) S_{r+1}=\operatorname{div}_{g}\left(P_{r}\left(\frac{\nabla_{1} f}{w}\right)\right),
$$

where div is $_{\text {is }}$ the divergence operator in the metric $g$.

Proof. First of all we recall that

$$
(r+1) S_{r+1}=\operatorname{trace}\left(A P_{r}\right)
$$

(cf. Lemma 2.1).

Let $\left\{e_{i}\right\}_{i=1}^{n}$ be as above. Then

$$
d x\left(e_{i}\right)=E_{i}+d f\left(e_{i}\right) E_{n+1} .
$$

We notice that if $\pi: M \times \mathbb{R} \longrightarrow M$ denotes the projection of $M \times \mathbb{R}$ into $M$, then $x^{-1}=\left.\pi\right|_{\Gamma_{f}}$. By the definition of $A$ we have

$$
A\left(e_{i}\right)=d x^{-1}\left(-\bar{\nabla}_{d x\left(e_{i}\right)} N\right)=d x^{-1}\left(-\bar{\nabla}_{E_{i}} N-d f\left(e_{i}\right) \bar{\nabla}_{E_{n+1}} N\right),
$$


but

$$
\begin{aligned}
\bar{\nabla}_{E_{n+1}} N & =\bar{\nabla}_{E_{n+1}}\left(\frac{\nabla_{1} f}{w}, 0\right)-\bar{\nabla}_{E_{n+1}}\left(\frac{E_{n+1}}{w}\right) \\
& =-\frac{1}{w} \bar{\nabla}_{E_{n+1}} E_{n+1}-E_{n+1}\left(\frac{1}{w}\right) E_{n+1}=0 .
\end{aligned}
$$

Therefore,

$$
\begin{aligned}
A\left(e_{i}\right) & =-d x^{-1}\left(\bar{\nabla}_{E_{i}} N\right)=-d x^{-1}\left(\bar{\nabla}_{E_{i}}\left(\frac{\nabla_{1} f}{w}, 0\right)+\bar{\nabla}_{E_{i}}\left(\frac{1}{w} E_{n+1}\right)\right) \\
& =-d x^{-1}\left(\left(\nabla_{1 e_{i}}\left(\frac{\nabla_{1} f}{w}\right), 0\right)+E_{i}\left(\frac{1}{w}\right) E_{n+1}\right) \\
& =-d x^{-1}\left(\nabla_{1 e_{i}}\left(\frac{\nabla_{1} f}{w}\right), E_{i}\left(\frac{1}{w}\right)\right) \\
& =-d \pi\left(\nabla_{1 e_{i}}\left(\frac{\nabla_{1} f}{w}\right), E_{i}\left(\frac{1}{w}\right)\right) \\
& =-\nabla_{1 e_{i}}\left(\frac{\nabla_{1} f}{w}\right) .
\end{aligned}
$$

Thus

$$
A(y)=-\nabla_{1 y}\left(\frac{\nabla_{1} f}{w}\right), \text { for all } y \in T(M)
$$

Hence, we have

$$
(r+1) S_{r+1}=\operatorname{trace}\left(A P_{r}\right)=-\operatorname{trace}\left(y \rightarrow P_{r} \nabla_{1 y}\left(\frac{\nabla_{1} f}{w}\right)\right),
$$

where we used that $P_{r}$ and $A$ commute (both have the same eigenvectors).

Proceeding with the proof of Lemma 3.1, we now claim that

$$
\operatorname{trace}\left(y \rightarrow P_{r} \nabla_{1 y}\left(\frac{\nabla_{1} f}{w}\right)\right)=\operatorname{trace}\left(y \rightarrow \nabla_{1 y}\left(P_{r}\left(\frac{\nabla_{1} f}{w}\right)\right)\right) .
$$

Lemma 3.2. If any of the following conditions

(i) $r=1$ and $\operatorname{Ric}_{(M, g)}=0$;

(ii) $r$ is arbitrary and $(M, g)$ is flat

holds, then

$$
\operatorname{trace}\left(y \rightarrow P_{r} \nabla_{1 y}(v)\right)=\operatorname{trace}\left(y \rightarrow \nabla_{1 y}\left(P_{r}(v)\right)\right), \text { for all } v \in T(M) .
$$

Proof of Lemma 3.2. First, we notice that using the definition of the curvature $R$ of $M$ and (3) we can see that

$$
\nabla_{1 y} A z-\nabla_{1 z} A y=A[y, z]+R(y, z)\left(\frac{\nabla_{1} f}{w}\right) .
$$

Suppose that (i) holds. Let us fix $p \in M$ and let $\left\{v_{i}\right\}_{i=1}^{n}$ be an orthonormal frame in a neighborhood of $p$ such that $\left\{v_{i}\right\}_{i=1}^{n}$ is geodesic at $p$, that is, $\nabla_{v_{i}} v_{j}(p)=0$ for $i, j \in\{1, \ldots, n\}$. It suffices to prove the lemma for $v=v_{j}, 1 \leq j \leq n$.

Since $\operatorname{trace}\left(y \rightarrow P_{1} \nabla_{y} v_{j}\right)(p)=\sum_{i}\left\langle v_{i}, P_{1} \nabla_{v_{i}} v_{j}\right\rangle(p)=0$, we have to show that

$$
\operatorname{trace}\left(y \rightarrow \nabla_{1 y} P_{1} v_{j}\right)(p)=0 .
$$


But

$$
\begin{aligned}
\operatorname{trace}\left(y \rightarrow \nabla_{1 y} P_{1} v_{j}\right)(p)= & \sum_{i=1}^{n}\left\langle v_{i}, \nabla_{1 v_{i}}\left(S_{1} v_{j}-A v_{j}\right)\right\rangle \\
= & \sum_{i=1}^{n}\left\langle v_{i}, v_{i}\left(S_{1}\right) v_{j}\right\rangle-\sum_{i=1}^{n}\left\langle v_{i}, \nabla_{1 v_{i}} A v_{j}\right\rangle \\
= & v_{j}\left(S_{1}\right)-\sum_{i=1}^{n}\left\langle v_{i}, \nabla_{1 v_{j}} A v_{i}\right\rangle \\
& +\sum_{i=1}^{n}\left\langle v_{i}, R\left(v_{j}, v_{i}\right) \frac{\nabla_{1} f}{w}\right\rangle \\
= & v_{j}\left(S_{1}\right)-\operatorname{trace}\left(y \rightarrow \nabla_{1 v_{j}}(A) y\right) \\
& +\operatorname{Ric} c_{(M, g)}\left(v_{j}, \frac{\nabla_{1} f}{w}\right) \\
= & v_{j}\left(S_{1}\right)-\operatorname{trace}\left(y \rightarrow \nabla_{1 v_{j}}(A) y\right),
\end{aligned}
$$

where in the third equality we used (5). Now, we claim that

$$
v_{j}\left(S_{r}\right)=\operatorname{trace}\left(y \rightarrow P_{r-1} \nabla_{1 v_{j}}(A) y\right) .
$$

We will prove (77) by making the computations in a basis $\left\{u_{i}\right\}_{i=1}^{n}$ that diagonalizes $A$. In such a basis, we have

$$
A=\operatorname{diagonal}\left(k_{1}, \ldots, k_{n}\right)
$$

and

$$
P_{r-1}=\operatorname{diagonal}\left(S_{r-1}\left(A_{1}\right), \ldots, S_{r-1}\left(A_{n}\right)\right)
$$

(see Lemma 2.1). Then, we have for $e \in T(M)$,

$$
\begin{aligned}
\operatorname{trace}\left(y \rightarrow P_{r-1} \nabla_{1 e}(A) y\right) & =\sum_{i=1}^{n}\left\langle u_{i}, P_{r-1} \nabla_{1 e}(A) u_{i}\right\rangle \\
& =\sum_{i=1}^{n} S_{r-1}\left(A_{i}\right)\left\langle u_{i}, \nabla_{1 e}\left(A u_{i}\right)-A \nabla_{1 e} u_{i}\right\rangle \\
& =\sum_{i=1}^{n} S_{r-1}\left(A_{i}\right) e\left(k_{i}\right)=\sum_{i=1}^{n} \frac{\partial S_{r}}{\partial k_{j}} e\left(k_{i}\right)=e\left(S_{r}\right),
\end{aligned}
$$

thus proving (7).

Now, suppose that (ii) holds. Then equation (5) becomes

$$
\nabla_{1 y} A z-\nabla_{1 z} A y=A[y, z]
$$

that is, the tensor $A$ is a "Codazzi tensor" in the metric $g$. Similarly to (i) and with the same notation, we have to prove that

$$
\operatorname{trace}\left(y \rightarrow \nabla_{1 y} P_{r} v_{j}\right)(p)=0
$$

and this we do by induction.

Since $P_{r}=S_{r} I-P_{r-1} A$, equation (9) holds provided that

$$
\operatorname{trace}\left(y \rightarrow \nabla_{1 y} P_{r-1} A v_{j}\right)(p)=\operatorname{trace}\left(y \rightarrow \nabla_{1 y}\left(S_{r} v_{j}\right)\right)(p) .
$$


Assuming that (4) holds for $r-1$ and using (77) and (8) we obtain

$$
\begin{aligned}
\sum_{i=1}^{n}\left\langle v_{i}, \nabla_{1 v_{i}} P_{r-1} A v_{j}\right\rangle & =\sum_{i=1}^{n}\left\langle v_{i}, P_{r-1} \nabla_{1 v_{i}} A v_{j}\right\rangle=\sum_{i=1}^{n}\left\langle v_{i}, P_{r-1} \nabla_{1 v_{j}} A v_{i}\right\rangle \\
& =\operatorname{trace}\left(y \rightarrow P_{r-1} \nabla_{1 v_{j}}(A) y\right)=v_{j}\left(S_{r}\right) \\
& =\operatorname{trace}\left(y \rightarrow \nabla_{1 y}\left(S_{r} v_{j}\right)\right)(p),
\end{aligned}
$$

which concludes the proof of Lemma 3.2.

Thus the claim is proved and by the definition of $d i v_{g}$, so is the Main Lemma.

For the case of graphs in $\mathbb{R}^{n}$, the above lemma was proved by $\mathrm{R}$. Reilly in $[\mathrm{Re}$.

Remark 3.3. The proof of Lemma 3.2 was inspired by the proof of equation (4.2) of [Ro], stating that

$$
\operatorname{trace}\left(u \rightarrow P_{r} \nabla_{u} v\right)=\operatorname{trace}\left(u \rightarrow \nabla_{u} P_{r} v\right) \text { for all } v \in T(M),
$$

when the ambient space has constant sectional curvature and $\nabla$ is the connection of the induced metric.

\section{Proof of Theorem 1.2 and Further Results}

Following [Cha, p.95] we define

Definition 4.1. Let $D \subset(M, g)$ be a domain. The Cheeger constant $\mathcal{H}(D)$ of $D$ is given by

$$
\mathcal{H}(D)=\inf _{\tilde{D}} \frac{\operatorname{vol}_{g}(\partial \tilde{D})}{\operatorname{vol}_{g}(\tilde{D})}
$$

where $\tilde{D} \subset D$ is a domain, $\operatorname{vol}_{g}(\tilde{D})$ is the volume of $\tilde{D}$ in the metric $g$ and $\operatorname{vol}_{g}(\partial \tilde{D})$ is the volume of $\partial \tilde{D}$ in the metric induced by $g$ in $\partial \tilde{D}$.

Suppose that $M$ is complete and noncompact. Let $p \in M$ and denote by $B_{p}(R) \subset$ $M$ the geodesic ball of center $p$ and radius $R$. We say that the volume of $M$ has polynomial growth if there exist positive numbers $\alpha, R_{0}$ and $a$ such that

$$
V\left(B_{p}(R)\right) \leq a R^{\alpha}, \text { for any } R \geq R_{0},
$$

where $V($.$) is the volume of the enclosed set.$

The next proposition is well known and since we could not find a suitable reference we give a sketch of the proof here for completeness.

Proposition 4.2. Suppose that $(M, g)$ is complete and that its volume has polynomial growth. Then

$$
\mathcal{H}(M)=0 .
$$

Proof. First, we observe that since the volume of $(M, g)$ has polynomial growth, $\lambda_{1}^{\Delta}(M)=0$ (see [CY, Proposition (9)]). Theorem (3) of [Cha] says that

$$
\lambda_{1}^{\Delta}(D) \geq \frac{\mathcal{H}^{2}(D)}{4}
$$


for any compact domain $D \subset M$, where $\lambda_{1}^{\Delta}(D)$ is the first eigenvalue of the Laplacian for $D$ in the Dirichlet problem. This implies that

$$
\lambda_{1}^{\Delta}(M) \geq \frac{\mathcal{H}^{2}(M)}{4}
$$

and then we conclude that $\mathcal{H}(M)=0$.

Proposition 4.3. Suppose that $M$ is flat, that the $r+1$-mean curvature of the graph $\Gamma_{f}$ satisfies $H_{r+1} \leq-k, k=$ constant $>0$, and that $\left\|P_{r}\right\|$ is bounded. Then we have

$$
(r+1)\left(\begin{array}{c}
n \\
r+1
\end{array}\right) k \leq \sup _{M}\left\|P_{r}\right\| \mathcal{H}(M)
$$

Proof. The Main Lemma 3.1 says that

$$
-(r+1) S_{r+1}=\operatorname{div}_{g}\left(P_{r}\left(\frac{\nabla_{1} f}{w}\right)\right) .
$$

By integrating this equation and by using Stokes Theorem we have, for a domain $D \subset M$,

$$
\begin{aligned}
-(r+1)\left(\begin{array}{c}
n \\
r+1
\end{array}\right) \int_{D} H_{r+1} d M & =-(r+1) \int_{D} S_{r+1} d M=\int_{D} \operatorname{div}_{g}\left(P_{r} \frac{\nabla_{1} f}{w}\right) d M \\
& =\int_{\partial D}\left\langle P_{r} \frac{\nabla_{1} f}{w}, \nu\right\rangle d s .
\end{aligned}
$$

Here, $\nu$ is the unit exterior vector field, normal to $\partial D$ and $d s$ is the element of volume of $\partial D$. Now, we use the hypotheses to obtain

$$
\begin{aligned}
(r+1)\left(\begin{array}{c}
n \\
r+1
\end{array}\right) \operatorname{kvol}_{g}(D) & \leq \int_{\partial D}\left\langle P_{r} \frac{\nabla_{1} f}{w}, \nu\right\rangle d s \leq \sup _{M}\left\|P_{r}\right\| \int_{\partial D}\left|\frac{\nabla_{1} f}{w}\right||\nu| d s \\
& \leq \sup _{M}\left\|P_{r}\right\| v_{g}(\partial D) .
\end{aligned}
$$

Theorem 4.4. Suppose that $(M, g)$ is flat and compact. Then there is no graph $\Gamma_{f}$ over $M$ (with orientation given by $N$ ) with $H_{r+1} \leq-k, k=$ constant $>0$.

Proof. By the definition of the Cheeger constant, we can see that $\mathcal{H}(M)=0$ when $M$ is compact. Also, the compactness of $M$ implies that $\left\|P_{r}\right\|$ is bounded. Suppose that there is such a graph. Then, by Proposition 4.3 we arrive at a contradiction.

Remark 4.5. Definition 4.1 is not the usual definition of the Cheeger constant for compact manifolds (without boundary). The idea of using the Cheeger constant in the present context was borrowed from [Sa].

Theorem 4.6. Suppose that $(M, g)$ is flat and complete. Then there is no graph $\Gamma_{f}$ over $M$ (with orientation given by $N$ ) with $\left\|P_{r}\right\|$ bounded and with $H_{r+1} \leq-k$, $k=$ constant $>0$.

Proof. Since $M$ is flat, its volume has polynomial growth. By Proposition 4.2 $\mathcal{H}(M)=0$. Suppose that there is such a graph. Then, by Proposition 4.3 we arrive at a contradiction. 
Theorem 1.2. There is no complete graph in $\mathbb{R}^{n+1}$ with $\left\|P_{r}\right\|$ bounded and negative $r+1$-mean curvature $H_{r+1}$ bounded away from zero, $r+1$ even.

Proof. We just take $M=\mathbb{R}^{n}$ in Theorem 4.6

In Proposition 4.3 and in Theorems 4.4 and 4.6, we used condition (ii) of Lemma 3.1. We can also use (i) of the same lemma to obtain the following results.

Proposition 4.7. Suppose that $\operatorname{Ric}_{M}=0$, that the scalar curvature of the graph $\Gamma_{f}$ satisfies $H_{2} \leq-k, k=$ constant $>0$, and that $\|A\|$ is bounded. Then

$$
2\left(\begin{array}{l}
n \\
2
\end{array}\right) k \leq \sup _{M}\left\|P_{1}\right\| \mathcal{H}(M)
$$

Theorem 4.8. Suppose that $\operatorname{Ric}_{M}=0$ and that $(M, g)$ is compact. Then there is no graph $\Gamma_{f}$ over $M$ with $H_{2} \leq-k, k=$ constant $>0$.

Theorem 4.9. Suppose that $\operatorname{Ric}_{M}=0$, that $(M, g)$ is complete and that its volume has polynomial growth. Then there is no graph $\Gamma_{f}$ with $\|A\|$ bounded and with $\mathrm{H}_{2} \leq-k, k=$ constant $>0$.

\section{REFERENCES}

[BC] J.L.Barbosa and A.G.Colares, Stability of hypersurfaces with constant r-mean curvature. Ann. Global Anal. Geom. 15, 277-297 (1997). MR 98h:52091

[Cha] I. Chavel, Eigenvalues in Riemmanian Geometry. Academic Press, Orlando, USA (1984). MR 86g:58140

[Che] S.S.Chern, On the curvatures of a piece of hypersurface in Euclidean space. Abh. Math. Sem. Univ. Hamburg 29, 77-91 (1965). MR 32:6376

[CY] S. Y. Cheng and S.T. Yau, Differential equations on Riemannian manifolds and their geometric applications. Comm. Pure Appl. Math 28, 333-354 (1975). MR 52:6608

[O] T.Okayasu, $O(2) \times O(2)$-Invariant hypersurfaces with constant negative scalar curvature in $E^{4}$. Proc. Amer. Math. Soc. 107, 4, 1045-1050 (1989). MR 90f:53094

[Re] R.C.Reilly, On the Hessian of a function and the curvatures of its graph. Michigan Math. J. 20, 373-383 (1973). MR 48:12364

[Ro] H.Rosenberg, Hypersurfaces of constant curvatures in space forms. Bull. Sc. Math. 117, 211-239 (1993). MR 94b:53097

[Sa] I.M.C.Salavessa, Graphs with parallel mean curvature. Proc. Amer. Math. Soc. 107, 2, 449-458 (1989). MR 90a:53072

[SX] B.Smyth and F.Xavier, Efimov's Theorem in dimension greater than two. Invent. Math. 90, 443-450 (1987). MR 89h:53014

Instituto de Matematica, UfRJ, Cx. Postal 68530, 21941-590 Rio de Janeiro, RJ, BRASIL

Current address: IMPA - Estrada Dona Castorina, 110, 22460-320 - Rio de Janeiro, RJ, Brasil

E-mail address: elbert@impa.br 